06.1

\title{
Магнитные характеристики нанокомпозита на основе ферритов висмута
}

\author{
() И.В. Плешаков, М.П. Волков, Н.А. Ломанова , Ю.И. Кузьмин, В.В. Гусаров \\ Физико-технический институт им. А.Ф. Иофффе РАН, Санкт-Петербург, Россия \\ ฯ E-mail: natus@mail.ioffe.ru
}

Поступило в Редакцию 6 июня 2020 г.

В окончательной редакции 27 июля 2020 г.

Принято к публикации 27 июля 2020 г.

\begin{abstract}
Изучены процессы намагничивания нанокомпозита $\mathrm{BiFeO}_{3}-\mathrm{Bi}_{2} \mathrm{Fe}_{4} \mathrm{O}_{9}$, полученного методом глициннитратного горения. Показано, что магнитные характеристики индивидуальных соединений $\mathrm{BiFeO}_{3}$ и $\mathrm{Bi}_{2} \mathrm{Fe}_{4} \mathrm{O}_{9}$ и композита существенно различаются. Новые свойства синтезированного вещества связываются с наличием обменного взаимодействия на границах раздела между фазами.
\end{abstract}

Ключевые слова: магнитные свойства, железосодержащие сложные оксиды висмута, нанокомпозиты.

DOI: 10.21883/PJTF.2020.21.50192.18414

Среди тех областей физики конденсированных сред, которые ориентированы на создание новых магнитных материалов, в последнее время выделилось направление, посвященное исследованию многофазных систем, состоящих из соединений с разным типом магнитного порядка [1-5]. Так, в работах $[1,2]$ сообщается о том, что нанокомпозиты на основе перовскитоподобного ортоферрита висмута $\mathrm{BiFeO}_{3}$ и муллитоподобного феррита $\mathrm{Bi}_{2} \mathrm{Fe}_{4} \mathrm{O}_{9}$ демонстрируют эффекты, связанные с обменным взаимодействием на границе раздела этих фаз. Вопросы об особенностях их формирования, природе взаимодействия и деталях механизма связи между их магнитными подсистемами сейчас широко обсуждаются в научной литературе.

Целью настоящей работы является проведение сравнительного анализа магнитных характеристик нанокомпозита $\mathrm{BiFeO}_{3}-\mathrm{Bi}_{2} \mathrm{Fe}_{4} \mathrm{O}_{9}$, отдельных соединений $\mathrm{BiFeO}_{3}$ и $\mathrm{Bi}_{2} \mathrm{Fe}_{4} \mathrm{O}_{9}$, а также их смеси, полученных описанным далее способом.

Образцы синтезировались методом глицин-нитратного горения с использованием в качестве исходных реагентов нитрата висмута, нитрата железа и глицина. Продукты горения подвергались термообработке последовательно при 350 и $550^{\circ} \mathrm{C}$, на каждом этапе в течение одного часа (аналогично методике, описанной в [6]). Соотношение реагентов подбиралось таким образом, чтобы получить либо индивидуальные соединения $\mathrm{BiFeO}_{3}$ и $\mathrm{Bi}_{2} \mathrm{Fe}_{4} \mathrm{O}_{9}$, либо состав $\mathrm{BiFeO}_{3}-\mathrm{Bi}_{2} \mathrm{Fe}_{4} \mathrm{O}_{9}$ с массовым соотношением $\mathrm{BiFeO}_{3}: \mathrm{Bi}_{2} \mathrm{Fe}_{4} \mathrm{O}_{9}=1: 1$. Кроме того, в этом же соотношении из индивидуальных соединений $\mathrm{BiFeO}_{3}$ и $\mathrm{Bi}_{2} \mathrm{Fe}_{4} \mathrm{O}_{9}$ была приготовлена механическая смесь. Далее для повышения качества кристаллизации все образцы дополнительно термообрабатывались при $650^{\circ} \mathrm{C}$ в течение $20 \mathrm{~min}$.

Фазовый состав этих материалов определялся на дифрактометре XRD-7000 Shimadzu ( $\mathrm{Cu} K_{\alpha}$-излучение). Рентгеновские дифрактограммы образцов композита и смеси представлены на рис. 1. В целом фазовый состав всех образцов соответствовал заданному синтезом. На дифрактограммах образцов композита и смеси фиксируются только рефлексы фаз $\mathrm{BiFeO}_{3}$ (PDF 14-181)

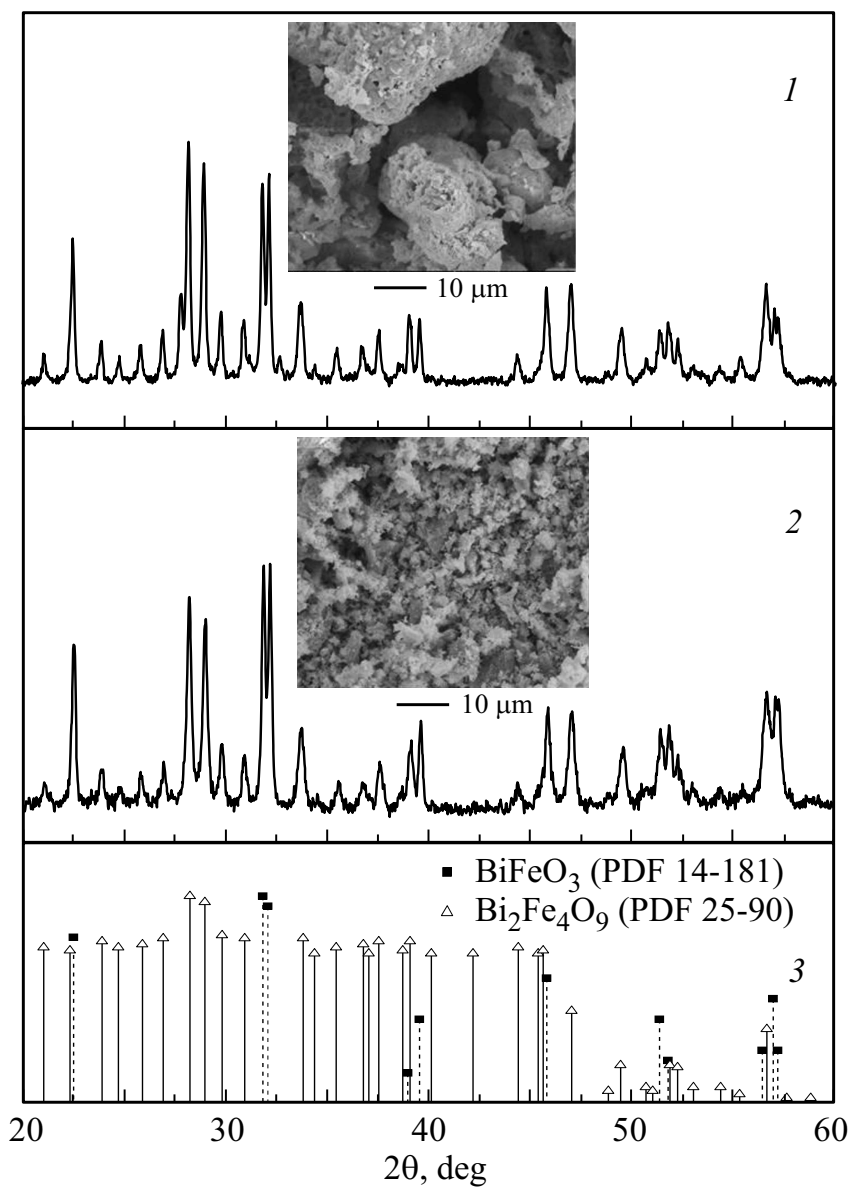

Рис. 1. Рентгеновские дифрактограммы композита (1) и смеси (2), штрих-диаграммы фаз $\mathrm{BiFeO}_{3}$ и $\mathrm{Bi}_{2} \mathrm{Fe}_{4} \mathrm{O}_{9}$ (3). На вставках приведены СЭМ-изображения. 
Размерные характеристики образцов

\begin{tabular}{c|c|c|c}
\hline \multirow{2}{*}{ Образец } & \multicolumn{2}{|c|}{$d, \mathrm{~nm}$} & \multirow{2}{*}{$D, \mu \mathrm{m}$} \\
\cline { 2 - 3 } & $\mathrm{BiFeO}_{3}$ & $\mathrm{Bi}_{2} \mathrm{Fe}_{4} \mathrm{O}_{9}$ & \\
\hline $\mathrm{Bi}_{2} \mathrm{Fe}_{4} \mathrm{O}_{9}$ & - & $60 \pm 3$ & $0.6 \pm 0.1$ \\
$\mathrm{BiFeO}_{3}$ & $70 \pm 2$ & - & $0.2 \pm 0.1$ \\
Смесь & $62 \pm 3$ & $37 \pm 4$ & $0.3 \pm 0.1$ \\
Композит & $70 \pm 2$ & $75 \pm 2$ & $1.0 \pm 0.1$
\end{tabular}

и $\mathrm{Bi}_{2} \mathrm{Fe}_{4} \mathrm{O}_{9}$ (PDF 25-90), параметры элементарных ячеек которых во всех образцах совпадают с литературными данными $[1,6,7]$. В образце на основе $\mathrm{BiFeO}_{3}$ были обнаружены следы примесей (например, $\mathrm{Bi}_{25} \mathrm{FeO}_{39}$, $\sim 5 \%$, не вносящих существенного вклада в величину полной намагниченности [3]. Средние размеры кристаллитов $d$ всех синтезированных образцов, определенные по формуле Шеррера, приведены в таблице, из которой видно, что они имеют небольшие различия, зависящие от фазового состава, что может быть связано с технологически обусловленными пространственными ограничениями роста.

Микроструктура и элементный состав исследовались методами сканирующей электронной микроскопии (СЭМ) и элементного энергодисперсионного микроанализа на сканирующем электронном микроскопе FEI Quanta 200 с приставкой EDAX. Было установлено, что порошки синтезированных веществ состоят из пористых агрегатов (см. вставки к рис. 1). Средний размер частиц $D$, определенный по СЭМ-изображениям образцов в программном комплексе ImageJ, приведен в таблице. В состав частиц, таким образом, входит значительное количество нанокристаллов (от нескольких десятков до нескольких тысяч). Анализ элементного состава по площади образцов показал, что в пределах погрешности метода соотношение элементов $\mathrm{Bi}: \mathrm{Fe}$ соответствует номинальному.

Магнитные измерения проводились с помощью вибрационного магнитометра PPMS Quantum Design, на котором для всех образцов были получены кривые намагничивания $M(H)(M-$ удельная намагниченность, $H$ - напряженность магнитного поля) при температуpax $T=5$ и $300 \mathrm{~K}$.

Результаты экспериментов для индивидуальных соединений, $\mathrm{BiFeO}_{3}$ и $\mathrm{Bi}_{2} \mathrm{Fe}_{4} \mathrm{O}_{9}$, показаны на рис. 2. Видно, что у $\mathrm{Bi}_{2} \mathrm{Fe}_{4} \mathrm{O}_{9}$ (рис. 2 , кривые 1 и 2) намагниченность значительно больше, чем у $\mathrm{BiFeO}_{3}$ (рис. 2, кривые 3 и 4), который, как известно, имеет пространственномодулированную антиферромагнитную спиновую структуру с периодом циклоиды $\lambda_{c} \approx 62 \mathrm{~nm}$ [8]. В нашем случае средний размер кристаллитов оказывается близким к критическому ( $d \sim \lambda_{c}$, см. таблицу $)$, т.е. циклоида должна частично разрушаться или искажаться. Как следствие, $\mathrm{BiFeO}_{3}$ демонстрирует лишь небольшой магнитный отклик, по интенсивности практически одинаковый при обеих температурах, что соответствует

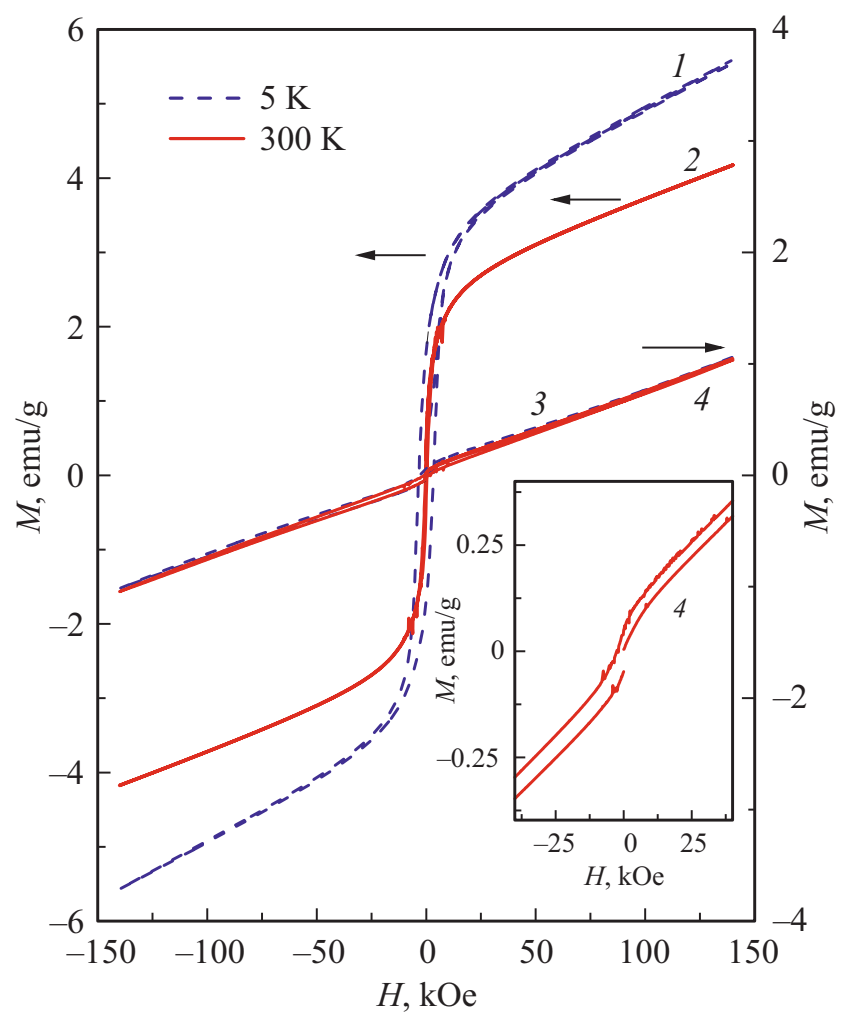

Рис. 2. Кривые намагничивания индивидуальных веществ $\mathrm{Bi}_{2} \mathrm{Fe}_{4} \mathrm{O}_{9}(1,2)$ и $\mathrm{BiFeO}_{3}(3,4)$. На вставке - особенности петли $M(H) \mathrm{BiFeO}_{3}$ в малых полях при $300 \mathrm{~K}$.

данным ряда других работ [3-6,9]. На вставке к рис. 2 приведена кривая $M(H)$ ортоферрита висмута в малых полях при $300 \mathrm{~K}$.

Согласно [10], магнетизм нанокристаллов $\mathrm{Bi}_{2} \mathrm{Fe}_{4} \mathrm{O}_{9}$ сильно зависит от $d$, причем считается, что объем кристаллитов упорядочивается антиферромагнитно (с достаточно высокой температурой Нееля), а моменты ионов оболочки образуют спиновое стекло. В нашем случае (рис. 2, кривые 1 и 2) кривые намагничивания этого образца при $T=5$ и $300 \mathrm{~K}$ подобны, что дает основание считать его магнитную структуру неизменяющейся в широком интервале температур (от гелиевой до комнатной). Наблюдавшееся здесь наибольшее значение намагниченности $M_{\max }$ (при $T=5 \mathrm{~K}$ и $\left.H=15 \mathrm{kOe}\right)$ существенно ниже намагниченности насыщения, рассчитанной для парамагнетика с известной для $\mathrm{Bi}_{2} \mathrm{Fe}_{4} \mathrm{O}_{9}$ концентрацией ионов $\mathrm{Fe}^{3+}$ в высокоспиновом состоянии с полностью замороженным орбитальным моментом, т.е. упорядочение этого вещества действительно предполагает наличие разориентированных спинов.

Как показывает рис. 3, кривые $M(H)$ смеси (рис. 3, кривые 1 и 2) и суммы кривых (рис. 3, кривые 1-1 и 2-1), определенных для индивидуальных соединений при 5 и $300 \mathrm{~K}$, практически совпадают (у смеси следует отметить только несколько увеличенное значение $M_{\max }$ ). Совершенно иной результат был получен для нанокомпозита (рис. 3, кривые 3 и 4). Видно, что при обеих 


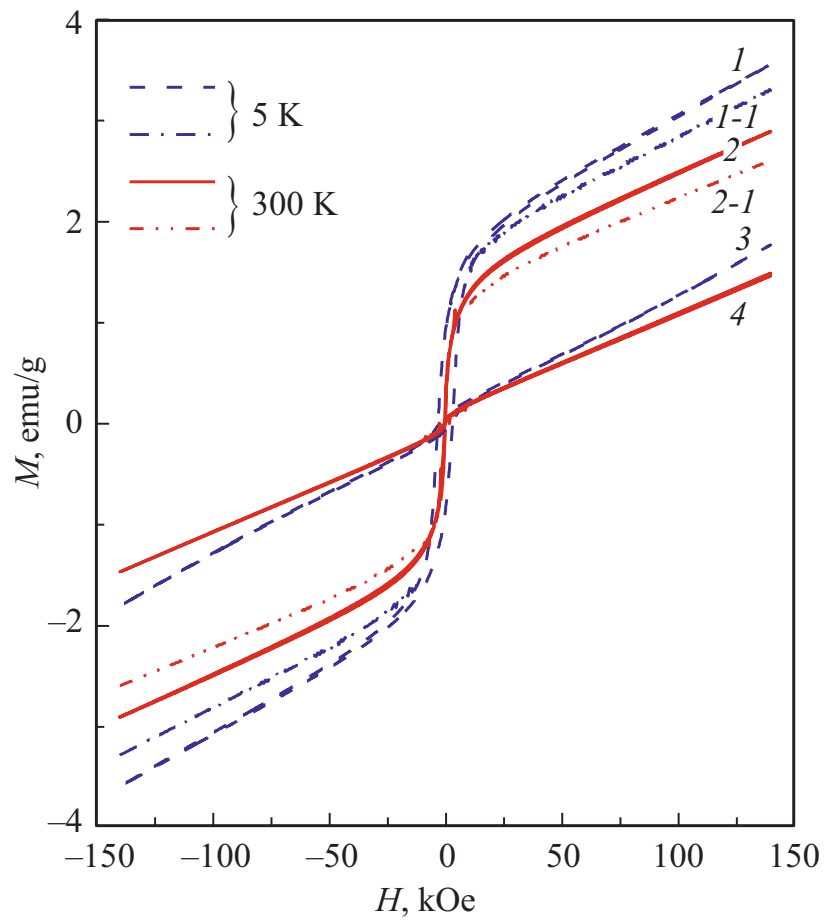

Рис. 3. Кривые намагничивания смеси $(1,2)$ и композита $(3,4)$. Кривые 1-1 и 2-1 получены сложением кривых $M(H)$ индивидуальных соединений с соответствующей весовой поправкой.

температурах кривые намагничивания этого образца функционально отличаются от соответствующих кривых смеси и обладают практически идеальным подобием кривым $M(H)$, зарегистрированным для $\mathrm{BiFeO}_{3}$ (рис. 2, кривые 3 и 4), но со значительно бо́льшим $M_{\max }$ (обратим внимание на характерный излом петли в малых полях). В качестве физического механизма, объясняющего данный эффект, может быть предложен имеющий обменную природу частичный перенос магнитного состояния от $\mathrm{BiFeO}_{3}$ на $\mathrm{Bi}_{2} \mathrm{Fe}_{4} \mathrm{O}_{9}$ через границу между ними. Предлагаемая модель аналогична использованной в [1], где рассматривалось взаимодействие между магнитными моментами $\mathrm{Bi}_{2} \mathrm{Fe}_{4} \mathrm{O}_{9}$ и локальными нескомпенсированными моментами на границе антиферромагнетика $\mathrm{BiFeO}_{3}$, опосредованное интерфейсом в виде спинового стекла. Различие состоит в том, что в [1] изучался материал, имеющий состав с небольшим содержанием $\mathrm{Bi}_{2} \mathrm{Fe}_{4} \mathrm{O}_{9}$ с кристаллитами значительно меньших размеров $d$, чем у кристаллитов $\mathrm{BiFeO}_{3}$. В нем также было зарегистрировано изменение магнитных характеристик, однако в нашем случае эффект взаимодействия проявился значительно сильнее и предположительно представлял собой распространение магнитного состояния одной из фракций на больший объем.

B нанокомпозите межфазный контакт происходит между нанообъектами (кристаллитами $\mathrm{BiFeO}_{3}$ и $\left.\mathrm{Bi}_{2} \mathrm{Fe}_{4} \mathrm{O}_{9}\right)$ с характерным размером $d$, что и определяет значительное изменение магнитного отклика данного материала (рис. 3, кривые 3 и 4). В противоположность этому в смеси соприкасаются преимущественно большие частицы с размерами $D$, и возникающее изменение $M(H)$ выражено очень слабо (рис. 3, кривые 1 и 2). Как уже отмечалось выше, в композитном материале кривые $M(H)$ строго подобны таким же кривым для $\mathrm{BiFeO}_{3}$ (рис. 2, кривые 3 и 4), но при разных $T$ они различаются по величине $M_{\max }$ (рис. 3 , кривые 3 и 4). Хотя конкретный механизм этого не вполне ясен, можно допустить, что параметры взаимодействия магнитных фракций зависят от температуры.

Качественная модель, принятая в работе для объяснения магнитных характеристик нанокомпозита $\mathrm{BiFeO}_{3}-\mathrm{Bi}_{2} \mathrm{Fe}_{4} \mathrm{O}_{9}$, конечно, пока остается гипотетической, однако явления, обнаруженные нами экспериментально, убедительно показывают, что данный материал, по крайней мере для случая использованной технологии изготовления и при сравнимом объеме составляющих его фаз, проявляет необычные магнитные свойства, интересные с точки зрения физики и, возможно, полезные для практики.

\section{Конфликт интересов}

Авторы заявляют, что у них нет конфликта интересов.

\section{Список литературы}

[1] Maity T., Goswami S., Bhattacharya D., Roy S. // Phys. Rev. Lett. 2013. V. 110. N 10. P. 107201.

[2] Maity T., Roy S. // J. Magn. Magn. Mater. 2020. V. 494. P. 165783.

[3] Ortiz-Quiñonez J.-L., Pal U., Villanueva M.S. // Inorg. Chem. 2018. V. 57. N 10. P. $6152-6160$. DOI: 10.1021/acs.inorgchem.8b00755

[4] Lomakin M.S., Proskurina O.V., Danilovich D.P., Panchuk V.V., Semenov V.G., Gusarov V.V. // J. Solid State Chem. 2020. V. 282. P. 121064. DOI: 10.1016/j.jssc.2019.121064

[5] Беспрозванных Н.В., Ершов Д.С., Синельщикова О.Ю. // ЖОХ. 2019. Т. 89. В. 12. C. $1955-1960$. DOI: $10.1134 \% 2 F S 1070363219120211$

[6] Lomanova N.A., Tomkovich M.V., Sokolov V.V., Ugolkov V.L., Panchuk V.V., Semenov V.G., Pleshakov I.V., Volkov M.P., Gusarov V.V. // J. Nanopart. Res. 2018. V. 20. N 2. P. 17. DOI: $10.1007 / \mathrm{s} 11051-018-4125-6$

[7] Almjasheva O.V., Lomanova N.A., Popkov V.I., Proskurina O.V., Tugova E.A., Gusarov V.V. // Nanosyst.: Phys. Chem. Math. 2019. V. 10. N 4. P. 428-437. DOI: $10.17586 / 2220-8054-2019-10-4-428-437$

[8] Sosnowska I., Peterlin-Neumaier T., Streichele E. // J. Phys. C. 1982. V. 15. N 23. P. 4835-4846. DOI: $10.1088 / 0022-3719 / 15 / 23 / 020$

[9] Ortiz-Quinonez J.L., Diaz D., Zumeta-Dube I., ArriolaSantamaría H., Betancourt I., Santiago-Jacinto P., NavaEtzana N. // Inorg. Chem. 2013. V. 52. N 18. P. 10306-10317. DOI: $10.1021 / \mathrm{ic} 400627 \mathrm{c}$

[10] Tian Z.M., Yuan S.L., Wang X.L., Zheng X.F., Yin S.Y., Wang C.H., Liu L. // J. Appl. Phys. 2009. V. 106. N 10. P. 103912. DOI: $10.1063 / 1.3259392$ 